comparisons across outcomes, these were standardised (difference between the individual score and the mean of all scores divided by the standard deviation, per reader, wave and time-point) before running the models. The higher the standardised coefficient the more change in inflammation/damage is captured.

Results: In total, 345 patients were included (mean (SD) symptom duration: 1.6 (0.9) years; $53 \%$ males; $89 \%$ HLA-B27 positive). Inflammation on MRI-SIJ (according to both the ASAS definition of sacroiliitis and the continuous SPARCC score) was more sensitive to change as compared to inflammation on the spine that remained essentially unchanged regardless of the outcome (table 1). Structural damage on the SIJ was found to increase over time, but with a higher standardised yearly rate of change on MRI-SIJ (range: $0.015-0.274$ ) as compared to XSIJ (range: $0.043-0.126$ ). Notably, $\geq 3$ Fatty lesions on MRI-SIJ was the structural outcome in the SIJ with highest sensitive to change $(0.274)$, while $\geq 3$ erosions was the least sensitive (0.015). Spine structural damage slowly progressed over time but, in contrast to SIJ, radiographic outcomes (i.e. $\geq 1$ syndesmophytes and mSASSS) were more sensitive to change than MRI structural outcomes.

Conclusions: Our data adds to the body of evidence showing that structural damage assessed in pelvic radiographs only has low sensitivity to change. MRI$\mathrm{SIJ}$ is a promising alternative (especially fatty lesions) capturing more structural changes. In contrast, in detecting structural change in early axSpA radiographic outcomes outperform MRI outcomes.

Disclosure of Interest: None declared

DOI: 10.1136/annrheumdis-2018-eular.1596

\section{FRI0166 CHARACTERISATION OF PHOSPHODIESTERASE 4 (PDE4) BLOCKADE IN THE SYNOVIUM OF PSORIATIC ARTHRITIS PATIENTS: A FOCUS ON SYNOVIAL INVASIVENESS AND T-CELL POLYFUNCTIONALITY}

S.M. Wade ${ }^{1}$, M. Canavan ${ }^{1}$, T. McGarry ${ }^{1}$, S.C. Wade ${ }^{1}$, D.J. Veale ${ }^{2}$, U. Fearon ${ }^{1}$. ${ }^{1}$ Molecular Rhuematology, Trinity College Dublin; ${ }^{2}$ Center for Arthritis and Rheumatic Diseases, St. Vincents University Hospital, Dublin, Ireland

Background: Owing to the multi-faceted nature of the pathogenesis of psoriatic arthritis (PsA), the development of multi-targeted agents has been an area of intensive research. Such agents include the phosphodiesterase 4 (PDE4) inhibitors, Rolipram and Apremilast, which elevate intracellular cAMP levels to modulate a number of anti-inflammatory mechanisms. However, the effect of PDE4 blockade within the complex inflammatory environment of the inflamed synovium remains to be elucidated.

Objectives: To characterise the effect of PDE4 blockade in PsA using ex vivo synovial whole tissue explants and synovial single cell suspensions reflective of the complex synovial micro-environment.

Methods : Ex-vivo PsA whole tissue synovial explants were cultured in the presence of PDE4 inhibitor, Rolipram, for 24 hour. The expression of pro-inflammatory mediators were quantified by ELISA and MSD multiplex. A 21 day synovial explant matrigel model was utilised to examine synovial fibroblast (SFC) invasiveness to allow for a long-term assessment. For the characterisation of synovial Tcells, synovial explants were digested and cultured in the presence of Rolipram for 8 hours, stimulated and stained for surface and intracellular T-cell markers. Cell surface expression of CD161 was used to identify Th17 lineage (CD161 +Th17 and exTh17 cells) or non-Th17 lineage (CD161-) Th1 cells. SPICE analysis was utilised to determine the proportions of mono- and polyfunctional T-cells, which were correlated with disease activity scores.

Results: Rolipram treatment inhibited the spontaneous secretion of inflammatory mediators IL-6, IL-8, MCP-1 and MMP-1 (all $p<0.05$ ), with a parallel increase in IL10 expression. Under DMSO control conditions, a significant increase in SFC outgrowths from PsA explants (indicative of SFC invasiveness) was observed from day 8-21 (all $p<0.05$ ), effects of which were significantly decreased in the presence of Rolipram (all $p<0.05$ ). A comparative analysis of T cells in PsA PBMC and synovial tissue revealed an enrichment of Th1 $(p<0.05)$, Th17 $(p=0.06)$ and exTh17 $(p<0.05)$ cells in PsA synovial tissue, which displayed distinct polyfunctional cytokine profiles, in particular Th17 cells, as compared to matched PBMC. The frequency of polyfunctional triple positive GM-CSF/TNF/L-17 and or IFNy producing Th1 $(r=0.8, p<0.05)$, Th17 $(r=0.6, p<0.16)$ and exTh17 $(r=0.9, p<0.05)$ cells positively correlated with PsA disease activity, suggesting an important role of T-cell polyfunctionality in PsA synovial pathogenesis. Analysis of synovial tissue cell suspensions and matched PBMC cultured in the presence of Rolipram showed a significant decrease in the proportion of these triple positivesynovial $\mathrm{T}$ cells compared to DMSO $(p<0.05)$, suggesting that PDE4 blockade can effectively targets the polyfunctional hyper-pathogenic synovial T cells in PsA, particularly polyfunctional CD $8+T$ cells and Th17 lineage, Th17 and exTh17 cells.
Abstract FRI0171 - Table 1. Standardised rate of change of imaging outcomes over 5 years of follow-up in early axSpA patients from the DESIR-cohort who fulfil the ASAS axSpA classification criteria.

\begin{tabular}{|c|c|c|}
\hline Imaging outcomes & $\begin{array}{c}\text { Baseline score* } \\
(\mathrm{N}=313-344)\end{array}$ & $\begin{array}{l}\text { Standardized rate } \\
\text { of change/ vear }\end{array}$ \\
\hline \multicolumn{3}{|l|}{$\begin{array}{l}\text { SACROILIAC JOINTS } \\
\text { Inflammatory lesions (MRI-SIJ) }\end{array}$} \\
\hline Sacroiliitis (ASAS criteria) & $134(39.2 \%)$ & -0.278 \\
\hline SPARCC SIJ score (0-72) & $4.7(7.9)$ & -0.441 \\
\hline \multicolumn{3}{|l|}{ Structural lesions (MRI-SIJ) } \\
\hline$\geq 5$ fatty lesion and / or erosions & $66(19.5 \%)$ & $0.238+t$ \\
\hline$\geq 3$ erosions & $60(17.7 \%)$ & 0.015 \\
\hline$\geq 3$ fatty lesions & $56(16.5 \%)$ & 0.274 \\
\hline Number of fatty lesions and/or erosions $(0-80)$ & $2.9(4.9)$ & 0.111 \\
\hline Number of erosions $(0-40)$ & $1.3(2.2)$ & 0.030 \\
\hline Number of fatty lesions $(0-40)$ & $1.5(3.5)$ & 0.140 \\
\hline Total structural lesions $¥(0-144)$ & $3.4(5.9)$ & 0.115 \\
\hline Total structural lesions without sclerosis (0-104) & $3.2(5.8)$ & 0.124 \\
\hline \multicolumn{3}{|l|}{ Structural lesions (X-SIU) } \\
\hline mNY dichotomous & $73(21.2 \%)$ & 0.044 \\
\hline mNY 1-grade change & NA & 0.126 \\
\hline mNY 1 -grade change and value $\geq 2$ & NA & 0.119 \\
\hline mNY continuous grade $(0-8)$ & $1.7(1.8)$ & 0.043 \\
\hline \multicolumn{3}{|l|}{ SPINE } \\
\hline \multicolumn{3}{|l|}{ Inflammatory lesions (MRI-Spine) } \\
\hline BME: $\geq 3$ lesions & $32(9.4 \%)$ & -0.032 \\
\hline BME: $\geq 5$ lesions & $19(5.6 \%)$ & -0.030 \\
\hline SPARCC Spine score (0-414) & $2.6(7.7)$ & -0.050 \\
\hline Berlin Spine score (0-69) & $0.9(2.7)$ & -0.055 \\
\hline \multicolumn{3}{|l|}{ Structural lesions (MRI-Spine) } \\
\hline$\geq 5$ fatty lesions & $5(1.6 \%)$ & -0.013 \\
\hline Total structural lesionst $(0-322)$ & $0.4(1.0)$ & 0.016 \\
\hline Number of fatty lesions (0-92) & $0.3(0.8)$ & 0.008 \\
\hline Number of corner erosions (0-92) & $0.1(0.2)$ & 0.012 \\
\hline Number of corner bone spurs $(0-92)$ & $0.1(0.3)$ & 0.027 \\
\hline \multicolumn{3}{|l|}{ Structural lesions (X-Spine) } \\
\hline$\geq 1$ syndesmophyte & $19(5.5 \%)$ & 0.037 \\
\hline mSASSS score (0-72) & $0.3(1.3)$ & 0.043 \\
\hline
\end{tabular}

Conclusions: PDE4 blockade mediates broad anti-inflammatory mechanisms in PsA synovial tissue through the reduced expression of pro-inflammatory mediators, decreased invasiveness and reduced T cell polyfunctionality. We also demonstrate the feasibility of using ex vivo models to determine "in situ like" assessments of therapeutic agents and further our understanding of disease pathogenesis.

Disclosure of Interest: None declared

DOI: 10.1136/annrheumdis-2018-eular.6952

\begin{tabular}{ll}
\hline FRI0167 & EXPRESSION LEVELS OF IL-17, IL-22 AND IL-23 \\
& RECEPTORS IN FOUR OSTEOBLAST MODELS AND \\
& THE EFFECTS OF IL-17, IL-22 AND IL-23 ON \\
& OSTEOBLASTS
\end{tabular}

J.-R. Zhang ${ }^{1}$, S.-M. Dai' ${ }^{2}{ }^{1}$ Department of Rheumatology and Immunology; ${ }^{2}$ Department of Rheumatology and Immunology, Shanghai Jiao Tong University Affiliated Sixth People's Hospital, Shanghai, China

Background: Ankylosing spondylitis (AS) is a chronic inflammatory joint disease that chiefly affects the sacroiliac joints and the spine. Radiographs reveal erosive changes at the corners of the vertebral bodies in the early stages of disease, and outgrowth of bony spurs known as syndesmophytes in the later stages. Some data imply a pivotal role of IL-23/IL-17 axis in the regulation of bone homeostasis However, it remains unknown whether IL-17, IL-22 or IL-23 has any direct effects on osteoblasts or new bone formation in AS.

Objectives: To examine the expressions of IL-17, IL-22, and IL-23 receptors in four osteoblast models and the effects of IL-17, IL-22, and IL-23 on osteoblasts. Methods: Gene expression levels of receptors, alkaline phosphatase (ALP), osteocalcin (OCN), and Runt-related transcription factor 2 (Runx-2), were 
evaluated by RT-PCR and real-time RT-PCR. Proliferative responses and cell cycle analysis were detected by a CCK-8 assay and flow cytometry, respectively. ALP activity and ALP mass were detected by an ALP activity assay and ALP staining, respectively.

Results: In primary osteoblasts, only the IL-17 receptor was expressed. In C2C12, MC3T3-E1, and Saos-2 cells, the genes of IL-17, IL-22, and IL-23 receptors were not detectable. None of IL-17, IL-22, and IL-23 had an obvious effect on the proliferation of primary osteoblasts, but IL-17 exhibited an inhibitory effect on the gene expression of ALP, OCN, and Runx-2. The ALP activity and ALP mass of primary osteoblasts were downregulated by IL-17 treatment in a dose-dependent manner.

Conclusions: Primary osteoblasts constitutively express IL-17 receptors, but none of $\mathrm{C} 2 \mathrm{C} 12$ cells, MC3T3-E1 cells, and Saos-2 cells express any receptors for IL-17, IL-22, and IL-23. IL-17 inhibits BMP-2-induced osteoblast differentiation. Disclosure of Interest: None declared

DOI: 10.1136/annrheumdis-2018-eular.1659

\section{FRI0172 INFLAMMATION ON MRI OF THE SACROILIAC JOINTS IS HIGHLY PREDICTIVE OF STRUCTURAL DAMAGE IN AXIAL SPONDYLOARTHRITIS PATIENTS IN CLINICAL PRACTICE: DATA FROM THE ASAS AND DESIR COHORTS}

A. Sepriano ${ }^{1}$, S. Ramiro ${ }^{1}$, R. Landewée ${ }^{2}$, M. Dougados ${ }^{3}$, D. van der Heijde ${ }^{1}$, M. Rudwaleit ${ }^{4}{ }^{1}{ }^{1}$ Rheumatology, Leiden University Medical Centre, Leiden; ${ }^{2}$ Rheumatology, Amsterdam Rheumatology and Clinical Immunology Center (ARC), Amsterdam, Netherlands; ${ }^{3}$ Rheumatology, Hôpital Cochin, Hôpitaux de Paris, Paris, France; ${ }^{4}$ Rheumatology, Klinikum Bielefeld, Bielefeld, Germany

Background: The effect of MRI-detected inflammation on the development of radiographic damage at the sacroiliac joints (SIJ) level in patients (pts) with axial spondyloarthritis (axSpA) has been previously shown when images were scored by trained central readers ${ }^{1}$. Central reading decreases measurement error, but does not translate easily to what is usually done in clinical practice.

Objectives: To test the possible effect of MRI-SIJ inflammation on structural damage in X-SIJ, when both are assessed by local readers as in daily clinical practice.

Methods: Pts with axSpA (according to the treating rheumatologist) from both the ASAS and DESIR cohorts were included. MRI-SIJ and X-SIJ were obtained at baseline (BL), and at follow-up (ASAS: mean 4.4 years; DESIR: 5 years) and scored by local readers (rheumatologists/radiologists). Images were taken unblinded to other imaging information and clinical characteristics. Readers had the option to view the baseline image when scoring the follow-up image. Bone Marrow Oedema (BME) at MRI-SIJ was assessed either without a formal definition (ASAS-cohort) or according to the ASAS definition (DESIR-cohort) as present/absent. Structural damage in the X-SIJ was defined according to the $\mathrm{mNY}$ criteria. The\% of structural net progression (number of 'progressors' minus the number of 'regressors' divided by the total number of pts) was assessed in subgroups according to CRP and BME status at BL. The effect of BME on MRISIJ on X-SIJ damage was evaluated in logistic regression models adjusted for potential confounders selected a priori on clinical grounds (gender, HLA-B27, CRP, symptom duration, variables available in both cohorts).

Results: In total, 150 (ASAS-cohort) and 421 (DESIR-cohort) pts had complete 5 year X-SIJ data available. Remarkably, but not unexpectedly, the\% of 'improvements in X-SIJ' was impressive both in the ASAS- and DESIR-cohorts $(10 \%$ and $5.7 \%$ respectively), yielding a total\% of net progression that was higher in the former than in the latter $(14.7 \%$ and $5.9 \%)$. Net progression in X-SIJ ranged from $0.0 \%$ to $33 \%$ and from $0 \%$ to $17.4 \%$ according to the presence of objective signs of inflammation at BL in the ASAS- and DESIR-cohorts, respectively (figure 1). In the multivariable analysis, the presence of baseline BME at MRI-SIJ both in the ASAS (OR=3.2 [95\% Cl: 1.3-7.9]), and DESIR cohorts (OR=7.4 [95\% Cl: 4.312.7]) was highly predictive of X-SIJ structural progression at follow-up (table 1).

Abstract FRI0172 - Table 1. Effect of inflammation on MRI-SIJ at baseline on X-SIJ structural damage at follow-up

\begin{tabular}{lc}
\hline Predictor Outcome & $\mathrm{mNY}$ \\
& OR $(95 \% \mathrm{Cl})$ \\
\hline Sacroiliitis on MRI-SIJ (ASAS-cohort) & $3.2(1.3 ; 7.9)^{*}$ \\
Sacroiliitis on MRI-SIJ (DESIR-cohort) & $\mathbf{7 . 4 ( 4 . 3 ; 1 2 . 7 )}$ \\
\hline${ }^{*}$ Adjusted for gender, HLA-B27, CRP, symptom duration. &
\end{tabular}

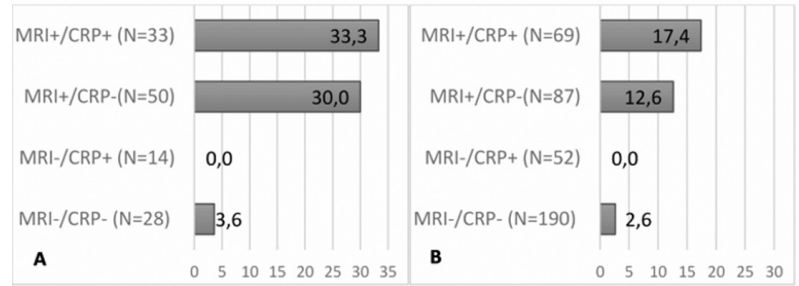

Abstract FRI0172 - Figure 1. Net progression from mNY-negative to mNY-positive according to baseline objective inflammatory markers in the ASAS (A) and DESIR (B) cohorts (ASAS: $\mathrm{N}=125 ; 25$ patients miss baseline MRI-SIJ; DESIR: $\mathrm{N}=398 ; 6$ patients miss baseline MRI-SIJ and 17 patients miss baseline CRP).

Conclusions: Our results, obtained in two independent cohorts, show that despite the expected increased 'noise' (measurement error) invoked by local reading, inflammation on $\mathrm{MRI-SIJ}$ still clearly predicts the later development of radiographic damage in axSpA.

\section{REFERENCE:}

[1] Dougados, et al. Ann Rheum Dis. 2017;76(11):1823-1828.

Disclosure of Interest: None declared

DOI: 10.1136/annrheumdis-2018-eular.2419

\section{FRI0173 1 IDENTIFICATION OF A TYPICAL PATTERN OF MRI LESIONS OF SACROILIAC JOINTS IN PATIENTS WITH OSTEITIS CONDENSANS ILII AS COMPARED TO AXIAL SPONDYLOARTHRITIS}

D. Poddubnyy ${ }^{1,2}$, N. Gobejishvili ${ }^{1}$, T. Diekhoff ${ }^{1}$, H. Weineck ${ }^{1}$, M. Llop ${ }^{1}$, V. Rios Rodriguez ${ }^{1}$, J. Sieper ${ }^{1}$, K.-G. Hermann ${ }^{1}$. ${ }^{1}$ Charité UniversitäTsmedizin Berlin; ${ }^{2}$ German Rheumatism Research Centre, Berlin, Germany

Background: Osteitis condensans ilii $(\mathrm{OCI})$ is regarded as a non-inflammatory disorder that is believed to be induced, for example, by mechanical stress and mechanical instability of the sacroiliac joints (SIJ) related to pregnancy/delivery. $\mathrm{OCl}$ is being increasingly recognised as an important differential diagnosis for axial spondyloarthritis (axSpA), due to onset at young age, possible inflammatory character of back pain and recently described presence of subchondral bone marrow oedema on magnetic resonance imaging (MRI) of the SIJ. So far, no systematic comparison of MRI changes in the sacroiliac joints in patients with $\mathrm{OCl}$ and axSpA has been performed.

Objectives: To compare active and chronic inflammatory lesions of the SIJ as detected by MRI in patients with $\mathrm{OCI}$ and axSpA

Methods: Using medical database search we identified $n=103$ patients aged $\geq 18$ years who were diagnosed with $\mathrm{OCl}$ upon presentation with chronic back pain in the Early Spondyloarthritis Clinic of the rheumatology department in the Charité University Hospital between January 2010 and May 2015. These patients were contacted in order to obtain an informed consent and to complete a survey on the disease-related history. A total of 27 patients had evaluable MRIs of the SIJ in STIR and T1-weighted sequences, which were used for the current study. These patients were matched to 27 patients with definite axSpA according to the back pain duration. MRIs were scored according to the Berlin scoring system for osteitis, fatty degeneration, erosions, sclerosis and ankylosis independently by 3 trained and calibrated readers who were blinded for all clinical data including diagnosis. In addition, the preferential localization of lesions (ventral, mid, or dorsal part of the SIJ) was recorded.

Results: There were no differences either in the osteitis score or in the proportion of patients with presence of osteitis on MRI of the SIJ between OCI and axSpA patients (table 1). The fatty degeneration score was significantly lower in $\mathrm{OCl}$ as compared to axSpA, although the difference in the prevalence of the fatty lesions did not reach the level of statistical significance. There was a non-significant trend towards a higher sclerosis score in $\mathrm{OCl}$ patients. Importantly, there was a highly significant difference in the erosion score and in the prevalence of erosions: only 2 (7.4\%) OCI vs. 18 (66.7\%) axSpA patients had at least one erosion (table 1). Importantly, none of the $\mathrm{OCl}$ patients had high-grade ( $>5$ erosions) erosive changes. 